Research Article

\title{
The Construction of Hilbert Spaces over the Non-Newtonian Field
}

\author{
Uğur Kadak ${ }^{1,2}$ and Hakan Efe ${ }^{1}$ \\ ${ }^{1}$ Department of Mathematics, Faculty of Sciences, Gazi University, 06500 Ankara, Turkey \\ ${ }^{2}$ Department of Mathematics, Faculty of Sciences and Arts, Bozok University, 66100 Yozgat, Turkey
}

Correspondence should be addressed to Uğur Kadak; ugurkadak@gmail.com

Received 23 January 2014; Revised 20 May 2014; Accepted 3 June 2014; Published 21 October 2014

Academic Editor: Seenith Sivasundaram

Copyright (C) 2014 U. Kadak and H. Efe. This is an open access article distributed under the Creative Commons Attribution License, which permits unrestricted use, distribution, and reproduction in any medium, provided the original work is properly cited.

\begin{abstract}
Although there are many excellent ways to present the principle of the classical calculus, the novel presentations probably lead most naturally to the development of the non-Newtonian calculi. In this paper we introduce vector spaces over real and complex nonNewtonian field with respect to the $*$-calculus which is a branch of non-Newtonian calculus. Also we give the definitions of real and complex inner product spaces and study Hilbert spaces which are special type of normed space and complete inner product spaces in the sense of $*$-calculus. Furthermore, as an example of Hilbert spaces, first we introduce the non-Cartesian plane which is a nonlinear model for plane Euclidean geometry. Secondly, we give Euclidean, unitary, and sequence spaces via corresponding norms which are induced by an inner product. Finally, by using the $*$-norm properties of complex structures, we examine CauchySchwarz and triangle inequalities.
\end{abstract}

\section{Introduction}

The foundation of the theory of Hilbert spaces was laid down in 1912, D. Hilbert (1862-1943), on integral equations. However, an axiomatic basis of the theory was provided by J. Von Neumann (1903-1957). Since then this topic has become one of the most interesting and powerful subjects. Moreover, Hilbert spaces are the simplest type of infinite dimensional Banach spaces to play a remarkable role in functional analysis.

Non-Newtonian calculus is an alternative to the usual calculus of Newton and Leibniz. It provides differentiation and integration tools based on non-Newtonian operations instead of classical operations. Every property in classical calculus has an analogue in non-Newtonian calculus. Generally, nonNewtonian calculus is a methodology that allows one to have a different look at problems which can be investigated via calculus. Furthermore the bigeometric calculus, which was created by Katz and Grossman in August 1970 as a branch of non-Newtonian calculus, has a derivative that is scale-free; that is, it is invariant under all changes of scales or units in function arguments and values. In some cases, for example, for wage-rate (in dollars, euro, etc.) related problems, the use of bigeometric calculus is advocated instead of a traditional Newtonian one (cf. [1-3]).

Bashirov et al. $[4,5]$ have recently concentrated on the multiplicative calculus and gave the results with applications corresponding to the well-known properties of derivatives and integrals in the classical calculus. Uzer [6] has extended the non-Newtonian calculus to the complex valued functions and was interested in the statements of some fundamental theorems and concepts of multiplicative complex calculus and demonstrated some analogies between the multiplicative complex calculus and classical calculus by theoretical and numerical examples. Çakmak and Başar [7] have studied the multiplicative differentiation for complex-valued functions and established the multiplicative Cauchy-Riemann conditions. Further, Tekin and Basar have introduced some certain sequence spaces over the non-Newtonian complex field by using *-calculus in [8] and many authors have introduced multiplicative calculus in biomedical image analysis and have derived non-Newtonian calculus as an alternative to the quantum calculus in $[9,10]$. Quite recently Kadak and Efe $[11,12]$ have determined duals and matrix transformations between certain sequence spaces over the non-Newtonian 
complex field. Also Çakmak and Başar have constructed certain spaces of functions over the field of non-Newtonian complex numbers [7]. Also Misirli and Gurefe [13] have introduced multiplicative Adams Bashforth-Moulton methods for differential equations.

The rest of the paper is organized as follows.

In Section 2, the systems of arithmetic which include $\alpha$ arithmetic and non-Newtonian norm and Banach spaces are established. Further, we give the corresponding results for the sequences of non-Newtonian real numbers concerning the convergent sequences of real numbers. In Section 3 we give the $*$-calculus and its applications which include some basic complex notions, that is, conjugate, modulus, and also series of complex terms. Furthermore, we define the nonNewtonian vector spaces corresponding to operations and we give the concept of $*$-basis, $*$-norm, and some necessary inequalities, that is, Minkowski and triangle inequality. Additionally we deduce the non-Cartesian geometry with related structures and give a relationship between some notions of Euclidean and non-Newtonian geometry. Section 4 is devoted to the construction of Hilbert spaces over real and complex field via related inner product in the sense of $*$ calculus. Finally, we introduce some applications that depend on complex inner product and examine some important inequalities, that is, Cauchy-Schwarz and triangle inequality.

\section{Preliminaries, Background, and Notation}

The system of complete ordered field evolved from the axiomatization of the real number system, whose basic ideas are well known. Informally, a complete ordered field is a system of a set $X$, four binary operations, $\dot{+}, \dot{-}, \dot{x}$ and for $X$, and an ordering relation $\dot{<}$ for $X$, all of which behave with respect to $X$ exactly as,,$+- \times, /,<$ behave with respect to the set of real numbers. We call $X$ the realm of the complete ordered field $[6$, p. 32]. A complete ordered field is called arithmetic if its realm is a subset of $\mathbb{R}$. A bijective function with domain $\mathbb{R}$ where its range is a subset of $\mathbb{R}$ is called a generator. For example, the identity function $I$, exponential function, and the function $x^{3}$ are generators. Each generator generates exactly one arithmetic, and conversely each arithmetic is generated by exactly one generator.

As a generator, we choose the function $\exp$ from $\mathbb{R}$ to the set $\mathbb{R}^{+}$of positive reals, that is to say that

$$
\begin{aligned}
\alpha: & \mathbb{R} \longrightarrow \mathbb{R}^{+} \\
x & \longmapsto \alpha(x)=e^{x}=y, \\
\alpha^{-1}: & \mathbb{R}^{+} \longrightarrow \mathbb{R} \\
& y \longmapsto \alpha^{-1}(y)=\ln y=x .
\end{aligned}
$$

If $I(x)=x$ for all $x \in \mathbb{R}$, then $I$ is called identity function whose inverse is itself. In the special cases $\alpha=I$ and $\alpha=\exp , \alpha$ generates the classical and geometric arithmetics, respectively. The set $\mathbb{R}(N)$ of non-Newtonian real numbers are defined as $\mathbb{R}(N):=\{\alpha(x): x \in \mathbb{R}\}$.

Consider any generator $\alpha$ - with range $A$. By $\alpha$-arithmetic, we mean the arithmetic whose domain is $A$ and whose operations are defined as follows: for $x, y \in \mathbb{R}$ and any generator $\alpha$,

$$
\begin{gathered}
\alpha \text {-addition } \quad x \dot{+} y=\alpha\left\{\alpha^{-1}(x)+\alpha^{-1}(y)\right\} \\
\alpha \text {-subtraction } \quad x \dot{y}=\alpha\left\{\alpha^{-1}(x)-\alpha^{-1}(y)\right\} \\
\alpha \text {-multiplication } \quad x \dot{\times} y=\alpha\left\{\alpha^{-1}(x) \times \alpha^{-1}(y)\right\} \\
\alpha \text {-division } \quad x / y, \frac{x}{y} N=\alpha\left\{\alpha^{-1}(x) \div \alpha^{-1}(y)\right\} \\
\alpha \text {-order } \quad x \dot{<} y \Longleftrightarrow \alpha^{-1}(x)<\alpha^{-1}(y) .
\end{gathered}
$$

Particularly if we choose $\alpha$-generator as exp, $\alpha(x)=e^{x}$ for $x \in \mathbb{R}$, then $\alpha^{-1}(x)=\ln x$ for $x \in \mathbb{R}^{+} ; \alpha$-arithmetic turns out to be Geometric arithmetic:

$$
\begin{aligned}
\alpha \text {-add. } x \dot{+} y=\alpha & \left\{\alpha^{-1}(x)+\alpha^{-1}(y)\right\}=e^{\{\ln x+\ln y\}}=x \cdot y \\
\alpha \text {-subt. } \quad x \dot{-} y & =\alpha\left\{\alpha^{-1}(x)-\alpha^{-1}(y)\right\}=e^{\{\ln x-\ln y\}} \\
& =x \div y, \quad y \neq 0 \\
\alpha \text {-mult. } \quad x \dot{\times} y & =\alpha\left\{\alpha^{-1}(x) \times \alpha^{-1}(y)\right\}=e^{\{\ln x \times \ln y\}} \\
& =x^{\ln y}=y^{\ln x} \\
\dot{\alpha} \text {-div. } \quad x / y & =\alpha\left\{\alpha^{-1}(x) \div \alpha^{-1}(y)\right\}=e^{\{\ln x \div \ln w\}} \\
& =x^{1 / \ln y}, \quad y \neq 1 .
\end{aligned}
$$

On the other hand, one can easily conclude that the summation turns out as follows:

$$
\begin{aligned}
& N \sum_{k=1}^{\infty} x_{k}=\alpha\left\{\sum_{k=1}^{\infty} \alpha^{-1}\left(x_{k}\right)\right\} \\
&=\alpha\left\{\alpha^{-1}\left(x_{1}\right)+\cdots+\alpha^{-1}\left(x_{k}\right)+\cdots\right\}, \\
& \forall\left(x_{1}, x_{2}, \ldots, x_{k}, \ldots\right) \in \mathbb{R} .
\end{aligned}
$$

The $\alpha$-positive real numbers, denoted by $\mathbb{R}^{+}(N)$, are the numbers $x$ in $\mathbb{R}$ such that $\dot{0} \dot{<} x$; the $\alpha$-negative real numbers, denoted by $\mathbb{R}^{-}(N)$, are those for which $x \dot{<} \dot{0}$. The $\alpha$-zero, $\dot{0}$, and the $\alpha$-one, $\dot{1}$, turn out to be $\alpha(0), \alpha(1)$, and $\alpha(-1)=\dot{-1}$. Thus the set of all $\alpha$-integers turn out to be the following:

$$
\mathbb{Z}(N)=\{\ldots, \alpha(-2), \alpha(-1), \alpha(0), \alpha(1), \alpha(2), \ldots\} .
$$

Theorem 1 (see $[14]) .(\mathbb{R}(N), \dot{+}, \dot{x})$ is complete field.

The $\alpha$-square of a number $x$ in $A \subset \mathbb{R}(N)$ is denoted by $x \dot{\times} x$ which will be denoted by $x^{2_{N}}$. For each $\alpha$-nonnegative number $t$, the symbol $\sqrt{x}^{N}$ will be used to denote $t=$ $\alpha\left\{\sqrt{\alpha^{-1}(x)}\right\}$ which is the unique $\alpha$-nonnegative number whose $\alpha$-square is equal to $x$, which means $t^{2}=x$. Throughout this section we denote the $p$ th non-Newtonian 
exponent and the $q$ th non-Newtonian root of $x \in \mathbb{R}(N)$ by $x^{p_{N}}$ and $\sqrt[q]{x^{N}}$, respectively. Therefore, we have

$$
\begin{aligned}
x^{2_{N}} & =x \dot{\times} x=\alpha\left\{\alpha^{-1}(x) \times \alpha^{-1}(x)\right\}=\alpha\left\{\left[\alpha^{-1}(x)\right]^{2}\right\} \\
x^{3_{N}} & =x^{2_{N}} \dot{\times} x=\alpha\left\{\alpha^{-1}\left\{\alpha\left[\alpha^{-1}(x) \times \alpha^{-1}(x)\right]\right\} \times \alpha^{-1}(x)\right\} \\
& =\alpha\left\{\left[\alpha^{-1}(x)\right]^{3}\right\} \\
& \vdots \\
x^{p_{N}} & =x^{(p-1)_{N}} \dot{\times} x=\alpha\left\{\left[\alpha^{-1}(x)\right]^{p}\right\} .
\end{aligned}
$$

The $\alpha$-absolute value of a number $x$ in $A \subset \mathbb{R}(N)$ is defined as $\alpha\left(\left|\alpha^{-1}(x)\right|\right)$ and is denoted by $|x|_{N}$. For each number $x$ in $A \subset \mathbb{R}(N),{\sqrt{x^{2}}}^{N}=|x|_{N}=\alpha\left(\left|\alpha^{-1}(x)\right|\right)$. Then we say

$$
|x|_{N}= \begin{cases}x, & x>\dot{0} \\ \dot{0}, & x=\dot{0}=\alpha\left\{\left|\alpha^{-1}(x)\right|\right\} \\ \dot{0}-x, & x<\dot{0} .\end{cases}
$$

For any numbers $r$ and $s$ in $A$, if $r \dot{<} s$, then the set of all numbers $x \in A$ such that $r \dot{<} x \dot{<} s$ which is called an $\alpha$-interval is denoted by $[r, s \dot{j}$. An $\alpha$-partition of an $\alpha$-interval $\dot{[} r, s]$ is any $\alpha$-progression whose first and last term are $r$ and $s$.

Let $\left(u_{n}\right)$ be an infinite sequence of the elements in $A \subset$ $\mathbb{R}(N)$. Then there is at most one element $u \in A$ such that every $\alpha$-interval with $u$ in its $\alpha$-interior contains all but finitely many terms of $\left(u_{n}\right)$. If there is such a number $u$, then $\left(u_{n}\right)$ is said to be $\alpha$-convergent to $u$, which is called the $\alpha$-limit of $\left(u_{n}\right)$. It is trivial that $\alpha=I ; \alpha$-convergence reduces to the classical convergence.

Proposition 2 (see [14]). For any $x, y \in \mathbb{R}^{+}(N)$, the following statements hold:

(i) $|x \dot{\times} y|_{N}=|x|_{N} \dot{x}|y|_{N}$;

(ii) $|x \dot{+} y|_{N} \dot{\leq}|x|_{N} \dot{+}|y|_{N}$ (triangle inequality);

(iii) let $p>1$ and $x_{k}, y_{k} \in \mathbb{R}^{+}(N)$ for $k \in\{1,2,3, \ldots, n\}$. Then,

$$
\sqrt[p]{N \sum_{k=1}^{n}\left(x_{k}+y_{k}\right)^{p_{N}}} \dot{N}{\sqrt[p]{N \sum_{k=1}^{n} x_{k}^{p_{N}}}}^{N}+\sqrt[p]{N \sum_{k=1}^{n} y_{k}^{p_{N}}}
$$

(Minkowski’s inequality).

Definition 3 (see [14]). Let $X$ be a nonempty set and let $d_{N}$ : $X \times X \rightarrow \mathbb{R}^{+}(N)$ be a function such that, for all $x, y, z \in X$, the following $\alpha$-metric axioms hold:

(NM1) $d_{N}(x, y)=\dot{0}$ if and only if $x=y$,

(NM2) $d_{N}(x, y)=d_{N}(y, x)$,

(NM3) $d_{N}(x, y) \dot{\leq} d_{N}(x, z) \dot{+} d_{N}(z, y)$.
Then, the pair $\left(X, d_{N}\right)$ and $d_{N}$ are called an $\alpha$-metric space and an $\alpha$-metric on $X$, respectively.

Corollary 4 (see $[14]) .\left(\mathbb{R}(N), d_{N}\right)$ is a non-Newtonian metric space.

Theorem 5 (see [14]). n-dimensional non-Newtonian Euclidian space $\mathbb{R}^{n}(N)$ consisting of all ordered $n$-tuples of nonNewtonian real numbers is a metric space with the metric $d_{N}$, defined by

$$
\begin{aligned}
d_{N}(x, y) & =\sqrt{N \sum_{k=1}^{n}\left(x_{k}-y_{k}\right)^{2_{N}}} \\
& =\alpha\left\{\sqrt{\sum_{k=1}^{n}\left[\alpha^{-1}\left(x_{k}\right)-\alpha^{-1}\left(y_{k}\right)\right]^{2}}\right\},
\end{aligned}
$$

where $x=\left(x_{1}, x_{2}, \ldots, x_{n}\right), y=\left(y_{1}, y_{2}, \ldots, y_{n}\right) \in \mathbb{R}^{n}(N)$.

Definition 6 (see [14]). Let $X$ be a vector space over the field $\mathbb{R}(N)$ and let $\|\cdot\|_{N}$ be a function from $X$ to $\mathbb{R}^{+}(N)$ satisfying the following non-Newtonian norm axioms: for $x, y \in X$ and $\alpha \in \mathbb{R}(N)$,

(NM1) $\|x\|_{N}=\dot{0} \Leftrightarrow x=\dot{0}$,

(NM2) $\|\alpha \dot{\times} x\|_{N}=|\alpha|_{N} \dot{\times}\|x\|_{N}$,

(NM3) $\|x \dot{+} y\|_{N} \dot{\leq}\|x\|_{N} \dot{+}\|y\|_{N}$.

Then, $\left(X,\|\cdot\|_{N}\right)$ is said to be a non-Newtonian normed space. It is trivial that a non-Newtonian norm $\|\cdot\|_{N}$ on $X$ defines a non-Newtonian metric $d_{N}$ on $X$ which is given by

$$
d_{N}(x, y)=\|x \dot{-} y\|_{N} ; \quad(x, y \in X),
$$

and it is called the non-Newtonian metric induced by the non-Newtonian norm.

Definition 7 (see [14]). (a) Convergent sequence: a sequence $\left(x_{n}\right)$ in a metric space $X=\left(X, d_{N}\right)$ is said to be convergent if for every given $\varepsilon>\dot{0}$ there exist an $n_{0}=n_{0}(\varepsilon) \in \mathbb{N}$ and $x \in X$ such that $d_{N}\left(x_{n}, x\right) \dot{<} \varepsilon$ for all $n>n_{0}$ and it is denoted by ${ }^{N} \lim _{n \rightarrow \infty} x_{n}=x$ or $x_{n} \stackrel{N}{\longrightarrow} x$, as $n \rightarrow \infty$.

(b) A sequence $\left(x_{n}\right)$ in a non-Newtonian metric space $X=\left(X, d_{N}\right)$ is said to be non-Newtonian Cauchy if for every $\varepsilon \dot{>} \dot{0}$ there is an $n_{0}=n_{0}(\varepsilon) \in \mathbb{N}$ such that $d_{N}\left(x_{n}, x_{m}\right) \dot{<} \varepsilon$ for all $m, n>n_{0}$.

Theorem 8 (see $[14]) .\left(\mathbb{R}^{n}(N), d_{N}\right)$ is complete.

Corollary 9 (see $[14]) . \mathbb{R}^{n}(N)$ is a Banach space with the norm $\|\cdot\|_{N}$ defined by

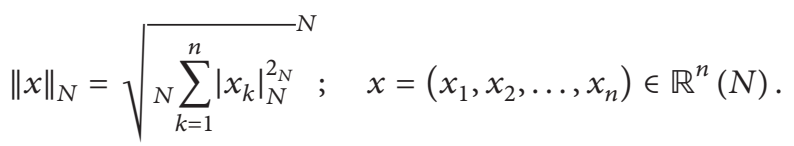




\section{3. "Star"-Calculus (*-Calculus) and Some Related Applications}

In the present section we give a new type of calculus denoted by *-calculus which represents general structure of nonNewtonian calculus. Since all arithmetics are isomorphic, one can obtain easily all arithmetics by using a unique function from alpha to the beta arithmetic.

Suppose that $\alpha$ and $\beta$ are two arbitrarily selected generators and ("star-") is also the ordered pair of arithmetics ( $\alpha$-arithmetic and $\beta$-arithmetic). The set $(B, \ddot{+}, \ddot{-}, \ddot{x}, \ddot{l})$ is a complete ordered field and $\beta$-generator generates $\beta$ arithmetic. Definitions given for $\alpha$-arithmetic are also valid for $\beta$-arithmetic. For example, $\beta$-convergence is defined by means of $\beta$-intervals and their $\beta$-interiors. In non-Newtonian calculus, $\alpha$-arithmetic is used for arguments and $\beta$-arithmetic is used for values; in particular, changes in arguments and values are measured by $\alpha$-differences and $\beta$-differences, respectively. The operators of this calculus type are applied only to functions with arguments in $A$ and values in $B$. The $*$-limit of a function with two generators $\alpha$ and $\beta$ is defined by

$$
\begin{gathered}
{ }^{*} \lim _{x \rightarrow a} f(x)=b \Longleftrightarrow \forall \varepsilon \ddot{>0}, \quad \exists \delta \dot{>} \dot{0} \ni|f(x) \ddot{-} b|_{N} \ddot{\leq} \varepsilon \\
\forall x \in A, \quad|x \dot{-} a|_{N} \dot{<} \delta .
\end{gathered}
$$

A function $f$ is $*$-continuous at a point $a$ in $A$ if and only if $a$ is an argument of $f$ and ${ }^{*} \lim _{x \rightarrow a} f(x)=f(a)$. When $\alpha$ and $\beta$ are the identity function $I$, the concepts of $*$-limit and $*$-continuity are identical with those of classical limit and continuity.

The isomorphism from $\alpha$-arithmetic to $\beta$-arithmetic is a unique function $\iota$ (iota) that obtains some required properties:

(i) $\iota$ is one to one;

(ii) $\iota$ is from $A$ onto $B$;

(iii) for any numbers $u$ and $v$ in $A$,

$$
\begin{aligned}
\iota(u \dot{+} v)=\iota(u) \ddot{+} \iota(v), & \iota(u \dot{-} v)=\iota(u) \ddot{-} \iota(v), \\
\iota(u \dot{\times} v)=\iota(u) \ddot{\times} \iota(v), & \iota(u \dot{\gamma} v)=\iota(u) \ddot{/} \iota(v) ; \\
v \neq \dot{0}, & u \dot{\leq} v \Longleftrightarrow \iota(u) \ddot{\leq} \iota(v) .
\end{aligned}
$$

It turns out that $\iota(x)=\beta\left\{\alpha^{-1}(x)\right\}$ for every $x$ in $A$ and that $\iota(\dot{n})=\ddot{n}$ for every integer $n$. Since, for example, $u \dot{+} v=$ $\iota^{-1}\{\iota(u) \ddot{+} \iota(v)\}$, it should be clear that any statement in $\alpha$ arithmetic can readily be transformed into a statement in $\beta$ arithmetic.

3.1. Non-Newtonian Complex Field with respect to the *-Calculus. Let $\alpha(a)=\dot{a} \in(A, \dot{+}, \dot{-}, \dot{x}, \dot{j})$ and $\beta(b)=$ $\ddot{b} \in(B, \ddot{+}, \ddot{-}, \ddot{x}, \ddot{l})$ be arbitrarily chosen elements from corresponding arithmetics. Then the ordered pair $(\dot{a}, \ddot{b})$ is called as a $*$-point. The set of all $*$-points is called the set of *-complex numbers and is denoted by $\mathbb{C}^{*}$; that is,

$$
\mathbb{C}^{*}:=\left\{z^{*}=(\dot{a}, \ddot{b}) \mid \dot{a} \in A, \ddot{b} \in B \text { and } A, B \subseteq \mathbb{R}\right\} .
$$

Defining the binary operations addition $\hat{+}$ and multiplication $\widehat{x}$ of $*$-complex numbers $z_{1}^{*}=\left(\dot{a}_{1}, \ddot{b}_{1}\right)$ and $z_{2}^{*}=\left(\dot{a}_{2}, \ddot{b}_{2}\right)$,

$$
\begin{aligned}
\hat{+}: \mathbb{C}^{*} \times \mathbb{C}^{*} & \longrightarrow \mathbb{C}^{*} \\
\left(z_{1}^{*}, z_{2}^{*}\right) & \longmapsto z_{1}^{*} \widehat{+} z_{2}^{*}=\left(\alpha\left\{a_{1}+a_{2}\right\}, \beta\left\{b_{1}+b_{2}\right\}\right) \\
= & \left(\dot{a}_{1}+\dot{a}_{2}, \ddot{b}_{1}, \ddot{b} \ddot{b}_{2}\right) \\
\hat{\times}: \quad \mathbb{C}^{*} \times \mathbb{C}^{*} \longrightarrow \mathbb{C}^{*} & \\
\left(z_{1}^{*}, z_{2}^{*}\right) \longmapsto z_{1}^{*} \widehat{\times} z_{2}^{*}= & \left(\alpha\left\{a_{1} a_{2}-b_{1} b_{2}\right\},\right. \\
& \left.\beta\left\{a_{1} b_{2}+b_{1} a_{2}\right\}\right),
\end{aligned}
$$

where $\dot{a}_{1}, \dot{a}_{2} \in A$ and $\ddot{b}_{1}, \ddot{b}_{2} \in B$.

Theorem 10 (see $[8]) .\left(\mathbb{C}^{*}, \widehat{+}, \widehat{x}\right)$ is a field.

Let $\ddot{b} \in B$. Then the number $\ddot{b} \ddot{\times} \ddot{b}$ is called the $\beta$-square and is denoted by $b^{2}$. Let $\ddot{b}$ be a nonnegative number in $B$. Then $\beta\left\{\sqrt{\beta^{-1}(\ddot{b})}\right\}$ is called the $\beta$-square root of $\ddot{b}$ and is denoted by $\sqrt{\ddot{b}}$. The $*$-distance $d^{*}$ between two arbitrarily elements $z_{1}^{*}=\left(\dot{a}_{1}, \ddot{b}_{1}\right)$ and $z_{2}^{*}=\left(\dot{a}_{2}, \ddot{b}_{2}\right)$ of the set $\mathbb{C}^{*}$ is defined by

$$
\begin{aligned}
d^{*}: & \left.\mathbb{C}^{*} \times \mathbb{C}^{*} \longrightarrow \ddot{[0}, \infty\right)=B^{\prime} \subset B \\
& \left(z_{1}^{*}, z_{2}^{*}\right) \longmapsto d^{*}\left(z_{1}^{*}, z_{2}^{*}\right) \\
& =\sqrt{\left[\iota\left(\dot{a}_{1}-\dot{a}_{2}\right)\right]^{\ddot{2}} \ddot{+}\left(\ddot{b}_{1}-\ddot{b_{2}}\right)^{2}} \\
& =\beta\left\{\sqrt{\left(a_{1}-a_{2}\right)^{2}+\left(b_{1}-b_{2}\right)^{2}}\right\} .
\end{aligned}
$$

Definition 11 (see [11] (complex conjugate)). Let $z^{*}=(\dot{a}, \ddot{b}) \epsilon$ $\mathbb{C}^{*}$. We define the non-Newtonian complex conjugate $\bar{z}^{*}$ of $z^{*}$ by $\bar{z}^{*}=\left(\alpha\{a\}, \beta\left\{-\beta^{-1}(\ddot{b})\right\}\right)=(\dot{a}, \ddot{-} \ddot{b})$. Conjugation changes the sign of the $*$-imaginary part of $z^{*}$ but leaves the real part the same. Thus

$$
\begin{gathered}
\mathscr{R} e\left(\bar{z}^{*}\right)=\mathscr{R e}\left(z^{*}\right)=\left(z^{*} \oplus \bar{z}^{*}\right) \dot{/} \dot{2}=\dot{a}, \\
\mathscr{I} m\left(\bar{z}^{*}\right)=\ddot{-} \mathscr{I} m\left(z^{*}\right)=\left(z^{*} \ominus \bar{z}^{*}\right) \ddot{/} \ddot{2}=\ddot{b} .
\end{gathered}
$$

Definition 12 (modulus). Consider $z^{*}=(\dot{a}, \ddot{b}) \in \mathbb{C}^{*}$. We define the $*$-modulus or $*$-absolute value of $z^{*}$ by $\ddot{\mid} z^{*} \mid=$ $\beta\left\{\left|z^{*}\right|\right\}=\beta\left\{\sqrt{a^{2}+b^{2}}\right\}$.

Proposition 13. Let $z^{*}=(\dot{a}, \ddot{b}), z_{1}^{*}=\left(\dot{a}_{1}, \ddot{b}_{1}\right)$, and $z_{2}^{*}=$ $\left(\dot{a}_{2}, \ddot{b}_{2}\right) \in \mathbb{C}^{*}$. Then the following results are obvious:

(i) $z_{1}^{*}=z_{2}^{*}$ if and only if $\overline{z_{1}^{*}}=\overline{z_{2}^{*}}$; 
(ii) $\overline{\overline{z^{*}}}=z^{*}$;

(iii) $\overline{z_{1}^{*} \widehat{ \pm} z_{2}^{*}}=\overline{z_{1}^{*}} \widehat{\underline{ \pm}} \overline{z_{2}^{*}}, \overline{z_{1}^{*} \widehat{\times} z_{2}^{*}}=\overline{z_{1}^{*}} \widehat{\times} \overline{z_{2}^{*}}$ and $\overline{z_{1}^{*} \widehat{/} z_{2}^{*}}=$ $\overline{z_{1}^{*} / z_{2}^{*}}$ where $z_{2}^{*} \neq \theta_{A}^{*}$ where $\theta_{A}^{*}=(\dot{0}, 00)$.

Definition 14 (see [11]). Given a sequence $\left(x_{k}\right)$ of nonNewtonian complex numbers, the formal notation

$$
* \sum_{k=1}^{\infty} x_{k}=x_{1} \widehat{+} x_{2} \widehat{+} x_{3} \widehat{+} \cdots \widehat{+} x_{n} \ldots
$$

is called an infinite non-Newtonian series with complex terms, or simply complex $N$-series. Also, for integers $n \in \mathbb{N}$, the finite $*$-sums $s_{n}=\sum_{*}^{n} \sum_{k=1}$ are called the partial sums of complex $N$-series. If the sequence $*$-converges to a complex number $s^{*}$ then we say that the series $*$-converges and write $s^{*}=\sum_{*}^{\infty} x_{n=1}$. The number $s^{*}$ is then called the $*$-sum of this series. If $\left(s_{n}\right) *$-diverges, we say that the series $*$-diverges, or that it is $*$-divergent.

3.2. Non-Newtonian Vector Space over the Field $\mathbb{F}^{*}$. We assume throughout this paper that $\mathbb{F}^{*}$ denotes either the nonNewtonian real field $\mathbb{R}(N)$ or the non-Newtonian complex field $\mathbb{C}^{*}$.

Definition 15. A non-Newtonian vector space ( $N$-vector space) over the field $F^{*}$ is a set $V$ on which two operations are defined, called $*$-addition and scalar $*$-multiplication, denoted + and $\times$ by

$$
\begin{array}{ll}
+: \quad V \times V \longrightarrow V \\
& (u, v) \longrightarrow u+v=\left(\dot{u}_{1} \dot{+} \dot{v}_{1}, \ddot{u}_{2} \ddot{+} \ddot{v}_{2}\right), \\
\times: \quad & F^{*} \times V \longrightarrow V \\
& (\lambda, u) \longrightarrow \lambda \times u=\left(\dot{\lambda} \dot{\times} \dot{u}_{1}, \ddot{\lambda} \ddot{\times} \ddot{u}_{2}\right),
\end{array}
$$

where the $*$-vectors are $u=\left(\dot{u}_{1}, \ddot{u}_{2}\right), v=\left(\dot{v}_{1}, \ddot{v}_{2}\right) \in X$ and the scalar $\lambda=(\dot{\lambda}, \ddot{\lambda}) \in \mathbb{F}^{*}$. Then the operations must satisfy the following conditions.

(i) Closure. For all $\lambda \in \mathbb{F}^{*}$ and all $u, v \in V$, the $*$-sum $u+v$ and the scalar $*$-product $\lambda \times v$ are uniquely defined and belong to $V$.

(ii) Associativity. For all $\xi, \eta \in F^{*}$ and all $u, v, w \in V$ then $u+(v+w)=(u+v)+w$ and $\xi \times(\eta \times v)=(\xi \times \eta) \times v$.

(iii) Additive Commutativity. For all $u, v \in V$ then $u+v=$ $v+u$.

(iv) Additive Identity. The set $V$ contains an additive *identity element, denoted by $\theta_{A}=(\dot{0}, 0)$, such that for all $u \in V, u+\theta_{A}=u$.

(v) Additive Inverse. The set $V$ contains an additive *inverse element, denoted by $u_{+}^{-1}=\left(\dot{-} \dot{u}_{1}, \ddot{-} \ddot{u}_{2}\right) \in V$, such that for all $u \in V, u+u_{+}^{-1}=u_{+}^{-1}+u=\theta_{A}$.

(vi) Multiplicative Identity. The set $V$ contains a multiplicative $*$-identity element, denoted by $\theta_{M}=(i, \ddot{0})$, such that for all $u \in V, u \times \theta_{M}=u$. (vii) Multiplicative Inverse. The set $V$ contains a multiplicative $*$-inverse element, denoted by $u_{\times}^{-1}=(\dot{m}, \ddot{n}) \in V$, where $\dot{m}=\alpha\left\{u_{1} /\left(u_{1}^{2}+u_{2}^{2}\right)\right\}$ and $\ddot{n}=\beta\left\{-u_{2} /\left(u_{1}^{2}+u_{2}^{2}\right)\right\}$ such that for all $u \in V, u \times u_{\times}^{-1}=u_{\times}^{-1} \times u=\theta_{M}$.

(viii) Distributive Laws. For all $\xi, \eta \in F^{*}$ and all $u, v \in V$ then $\xi \times(u+v)=(\xi \times u)+(\xi \times v)$ and $(\xi+\eta) \times u=$ $(\xi \times u)+(\eta \times u)$.

Definition 16. Let $S=\left\{v_{1}, \ldots, v_{n}\right\}$ be a set of $*$-vectors in $N$ vector space $V$ over the field $\mathbb{F}^{*}$. Any $*$-vector of the form $v$ by $v=\left(\lambda_{1} \widehat{\times} v_{1}\right) \hat{+}\left(\lambda_{2} \widehat{\times} v_{2}\right) \hat{+} \cdots \hat{+}\left(\lambda_{n} \widehat{\times} v_{n}\right), \lambda_{n} \in \mathbb{F}^{*}$ for all $n \in \mathbb{N}$, is called a linear combination of the $*$-vectors in $S$. The set $S$ is said to $*$-span $V$ if each element of $V$ can be expressed as a linear combination of the $*$-vectors in $S$.

Definition 17. Let $S=\left\{v_{1}, \ldots, v_{n}\right\}$ be a set of $*$-vectors in $N$-vector space $V$ over the field $\mathbb{F}^{*}$. Then the following statements hold.

(i) The *-vectors in $S$ are said to be linearly dependent if one of the $*$-vectors can be expressed as a linear combination of the others. If not, then $S$ is said to be a linearly independent set.

(ii) A subset of $N$-vector space $V$ is called a $*$-basis for $V$ if it *-spans $V$ and is linearly independent.

(iii) If $V$ has a finite $*$-basis, then it is said to be finite dimensional, and the number of $*$-vectors in the $*$ basis is called the dimension of $V$.

A $N$-vector space $V$ is said to be finite-dimensional if there exists a finite subset of $V$ which is a $*$-basis of $V$. If no such finite subset exists, then $V$ is said to be infinite-dimensional.

Now, we define the norm function and derive some required inequalities in the sense of non-Newtonian complex calculus.

Definition 18. Let $X$ be a $N$-vector space over the field $\mathbb{C}^{*}$ and let $\ddot{\|} \cdot \ddot{\|}$ be a function from $X$ to $\mathbb{R}_{\beta}^{+}(N)=\{\beta(x): x \in \mathbb{R}\}$ satisfying the following non-Newtonian norm axioms: for $x$, $y \in X$ and $\lambda \in \mathbb{C}^{*}$,

(N1) $\ddot{\|} x \ddot{\|}=\ddot{0} \Leftrightarrow x=\theta_{C}^{*}\left(\theta_{C}^{*}=\{(\dot{0}, \ddot{0}),(\dot{0}, \ddot{0}), \ldots,(\dot{0}, \ddot{0})\}\right.$,

(N2) $\ddot{\|} \lambda \widehat{\times} x \ddot{\|}=\ddot{\mid} y \mid \ddot{x} \ddot{\|} x \ddot{\|}(\ddot{\mid} y \mid$ is $*$-complex modulus),

(N3) $\ddot{\|} x \hat{+} y \ddot{\|} \ddot{\leq} \ddot{\|} \ddot{\|} \ddot{+} \ddot{\|} y \ddot{\|}$.

Then, $(X, \ddot{\|} \cdot \ddot{\|})$ is said to be a $*$-normed space. It is trivial that a $*$-norm $\ddot{\|} \cdot \ddot{\|}$ on $X$ defines a $*$-metric $d^{*}$ on $X$ which is given by $d^{*}(x, y)=\ddot{\|} x=y \ddot{\|} ;(x, y \in X)$ and is called the $*$-metric induced by the $*$-norm.

In other words, $\ddot{\|} z^{*} \|=d^{*}\left(z^{*}, \theta^{*}\right)=$ $\sqrt{[\iota(\dot{a}-\dot{0})]^{2}+(\ddot{b} \ddot{-} \ddot{0})^{\ddot{2}}}=\beta\left\{\sqrt{a^{2}+b^{2}}\right\}$ where $z^{*}=(\dot{a}, \ddot{b})$ and $\theta^{*}=(\dot{0}, \ddot{0})$. Moreover, for all $z_{1}{ }^{*}, z_{2}{ }^{*} \in \mathbb{C}^{*}$ we have $d^{*}\left(z_{1}^{*}, z_{2}^{*}\right)=\ddot{\|} z_{1}^{*}=z_{2}^{*} \ddot{\|}$. 
Lemma 19 (see [8]). Let $z_{1}^{*}, z_{2}^{*} \in \mathbb{C}^{*}$. Then the following statements hold:

(i) $\ddot{\|} z_{1}^{*} \uparrow z_{2}^{*} \ddot{\|} \ddot{\leq} \ddot{\|} z_{1}^{*} \ddot{\|} \ddot{+} \ddot{\|} z_{2}^{*} \ddot{\|}$, (*-triangle inequality);

(ii) $\ddot{\|} z_{1}^{*} \widehat{\times} z_{2}^{*} \ddot{\|}=\left\|\ddot{\|} z_{1}^{*}\right\| \ddot{x} \ddot{\|} z_{2}^{*} \ddot{\|}$.

Theorem 20 (see $[8]) .\left(\mathbb{C}^{*}, d^{*}\right)$ is a complete metric space.

Corollary 21 (see [8]). $\mathbb{C}^{*}$ is a Banach space with the *-norm $\ddot{\|} \cdot \ddot{\|}$ defined by

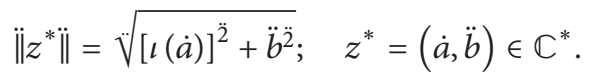

Remark 22 (see $[7$, p. 9]). Let $\alpha$ and $\beta$ be two generator functions and $z^{*} \in \mathbb{C}^{*}$. Then $z^{*} \widehat{\times} \overline{z^{*}}=\left|\ddot{\mid} z^{*}\right|^{\ddot{2}}$ holds.

At the final stage of this section, following Tekin and Basar [8], we give the definitions of some classical sets of sequences over the complex field $\mathbb{C}^{*}$ with respect to the $*$-calculus. That is to say that

$$
\begin{aligned}
& \omega^{*}=\left\{z^{*}=\left(z_{k}^{*}\right): z_{k}^{*} \in \mathbb{C}^{*} \forall k \in \mathbb{N}\right\}, \\
& \ell_{\infty}^{*}=\left\{z^{*}=\left(z_{k}^{*}\right) \in \omega^{*}: \sup _{k \in \mathbb{N}} \ddot{\|} z_{k}^{*} \ddot{\|}<\infty\right\}, \\
& c^{*}=\left\{z^{*}=\left(z_{k}^{*}\right) \in \omega^{*}: \exists l \in \mathbb{C}^{*} \ni{ }^{*} \lim _{k \rightarrow \infty} z_{k}^{*}=l\right\}, \\
& c_{0}^{*}=\left\{z^{*}=\left(z_{k}^{*}\right) \in \omega^{*}:{ }_{k \rightarrow \infty}^{*} \lim _{k}^{*}=\theta^{*}\right\}, \\
& \ell_{p}^{*}=\left\{z^{*}=\left(z_{k}^{*}\right) \in \omega^{*}:{ }_{*}^{\infty} \ddot{\|} z_{k}^{*} \ddot{\|}^{\ddot{p}}<\infty\right\}, \quad(1 \leq p<\infty) .
\end{aligned}
$$

3.3. Non-Cartesian Geometry. Classical calculus and Cartesian analytic geometry are based on classical arithmetic, which is usually called the real number system. But it was use nonclassical arithmetics that led to the general theory of the non-Newtonian calculi, to the development of nonCartesian analytic geometries, which is a nonlinear model for plane Euclidian geometry, to the creation of a new theory of subjective probability, and to the conception of new kinds of vectors, centroids, least-square methods, and complex numbers. Within non-Cartesian geometry, which is an arithmetic model for Euclidian geometry, one can define counterparts of all Euclidian notions, some of which will be discussed in this section.

Definition 23 (see [2]). Let $p$ be a $\beta$-positive real valued function. The $*$-slope of $p$ is its $\beta$-change over any $\alpha$-interval of $*$-extent 1 . For example, $*$-slope of the (iota) function $\iota^{-1}\{(m \dot{\times} x) \dot{+} c\}$ turns out to be $\iota(m)$. In particular, the $*$-slope of $\iota$ equals $\ddot{m}$.

Definition 24 (see [2]). By *-plane one means plane that is ruled off in squares and labeled as follows: the horizontal axis is marked $\alpha$-integers $\ldots, \alpha(-1), \alpha(0), \alpha(1), \ldots$ which equals $\ldots, \dot{-2}, \dot{-1}, \dot{0}, \dot{1}, \dot{2}, \ldots$, but on the vertical the equispaced points are marked with the $\beta$-integers $\ldots, \beta(-2), \beta(-1), \beta(0), \beta(1), \beta(2), \ldots$, which equals $\ldots, \ddot{-} \dot{2}$,

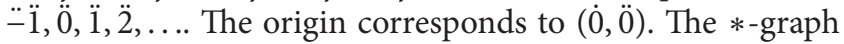
of a set of $*$-points is the result of plotting them on the $*$ plane.

For example, if $\alpha=I$ and $\beta=\exp$, then $*$-plane is semilog paper that is logarithmically scaled on the vertical axis.

Definition 25 (see [2]). A $*$-line is a set $L$ of at least two distinct $*$-points such that, for any distinct $*$-points $P_{1}$ and $P_{2}$ in $L$, an *-positive point $P_{3}$ is in $L$ if and only if $P_{1}, P_{2}$, and $P_{3}$ are $\alpha$-collinear.

\section{Theorem 26 (see [2]). The following statements hold.}

(a) Let the function $\beta$ be an $\exp$ generator. Then the class of nonvertical *-lines is identical with the class of power functions.

(b) The nonvertical *-lines are *-parallel if and only if they are identical or have no common point. For example, the $*$-lines with equations $y=x^{2}$ and $y=2 x^{2}$, where $x>0$, are $*$-parallel.

(c) The nonvertical $*$-lines are $*$-parallel if and only if they have the same *-slope.

(d) The nonvertical $*$-lines are $*$-perpendicular if and only if the product of their *-slope is $-\ddot{1}$.

\section{Main Results}

In the present section we give two definitions about real and complex inner product spaces with non-Newtonian calculus, respectively. For the real case we use only an alpha generator corresponding to the operations addition $(\dot{+})$ and multiplication $(\dot{x})$. Furthermore for complex case we use two generator alpha and beta corresponding to the operations complex addition $\widehat{+}$ and complex multiplication $\widehat{x}$.

Definition 27. A non-Newtonian real inner product (real $N$ inner product) space is a real $N$-vector space $X$ with a real $N$ inner product defined on $X$. A non-Newtonian Hilbert space over the field $\mathbb{R}(N)$ is a complete $N$-inner product space. Here, a $N$-inner product on $X$ is mapping of $X \times X$ into the scalar real field $\mathbb{R}(N)$ of $X$; that is, with every pair of $\alpha$-vectors $x$ and $y$ there is associated scalar which is written $\langle\cdot, \cdot \dot{\rangle}$ and is called the real $N$-inner product of $x$ and $y$, such that, for all $\alpha$-vectors, $x, y, z \in X$ and $\lambda, \mu \in \mathbb{R}(N)$, one has the following:

(RIP1) $\langle x \dot{+} y, z\rangle=\dot{\langle} x, z\rangle \dot{\psi} \dot{\langle} y, z\rangle$;

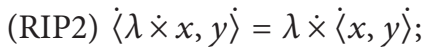

(RIP3) $\dot{\langle x}, y\rangle=\dot{\langle} y, x\rangle$;

(RIP4) $\dot{\langle} x, x\rangle \dot{0} \dot{0}$ and $\dot{\langle}, x\rangle=\dot{0}$ if and only if $x=\theta_{\alpha}$ for $\theta_{\alpha}=(\dot{0}, \dot{0}, \ldots, \dot{0})$. 
A real $N$-inner product on $X$ defines an $\alpha$-norm on $X$ given by

$$
\|x\|_{N}=\sqrt{\dot{\langle x, x\rangle}}^{N}
$$

and a non-Newtonian metric on $X$ is given by

$$
d_{N}(x, y)=\|x-y\|_{N}=\sqrt{\langle x-y, x-y\rangle}^{N} .
$$

Hence all real $N$-inner product spaces are $N$-normed spaces, and all Hilbert spaces are Banach spaces in non-Newtonian means.

Definition 28. A non-Newtonian complex inner product (complex $N$-inner product) space is a complex $N$-vector space $X$ with a complex $N$-inner product defined on $X$. Here, a $N$-inner product on $X$ is mapping of $X \times X$ into the scalar complex field $\mathbb{C}^{*}$ of $X$; that is, with every pair of $*$-vectors $x$ and $y$, there is associated scalar which is written $\ddot{\langle} \cdot, \cdot \ddot{\rangle}$ and is called the complex $N$-inner product of $x$ and $y$, such that for all $*$-vectors, $x, y, z \in X$ and $\lambda^{*}, \mu^{*} \in \mathbb{C}^{*}$, one has the following:

(CIP1) $\ddot{\langle} x \widehat{+} y, z \ddot{\rangle}=\ddot{\langle} x, z \ddot{\rangle} \hat{+} \ddot{\langle} y, z \ddot{\rangle}$;

(CIP2) $\ddot{\langle} \lambda^{*} \widehat{\times} x, y \ddot{\rangle}=\lambda^{*} \widehat{\times} \ddot{\langle} x, y \ddot{\gamma}$;

(CIP3) $\ddot{\langle} x, y \ddot{\rangle}=\overline{\langle} y, x \ddot{\rangle}$;

(CIP4) $\ddot{\langle} x, x \ddot{\rangle}=\ddot{0}$ if and only if $x=\theta_{C}^{*}$.

A complex $N$-inner product on $X$ defines a $*$-norm on $X$ given by

$$
\ddot{\|} x \|=\sqrt{\ddot{\langle x, x\rangle}}
$$

and a non-Newtonian metric on $X$ is given by

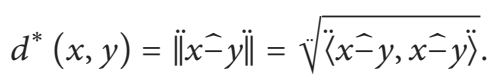

In (CIP3), the bar denotes complex conjugation in Definition 11. The proof that the inclusion (24) satisfies the axioms (N1) to (N4) of a *-norm will be given later. Additionally, from (CIP1) and (CIP3) we obtain the following formulas:
(a) $\ddot{\langle} \lambda^{*} \hat{x} x \hat{+} \mu^{*} \hat{x} y, z \ddot{\rangle}=\lambda^{*} \hat{x} \ddot{\langle} x, z \ddot{\rangle} \hat{+} \mu^{*} \hat{x} \ddot{\langle} y, z \ddot{\rangle}$;
(b) $\ddot{\langle} x, \lambda^{*} \hat{\times} y \ddot{\rangle}=\overline{\lambda^{*}} \hat{\times} \ddot{\langle} x, y \ddot{\rangle}$;
(c) $\left.\ddot{\langle} x, \lambda^{*} \hat{\times} y \widehat{+} \mu^{*} \hat{x} z \ddot{\rangle}=\overline{\lambda^{*}} \hat{x} \ddot{\langle} x, y \ddot{\gamma} \widehat{+} \overline{\mu^{*}} \hat{\times} \ddot{\langle} x, z\right\rangle$,

for all $\lambda^{*}, \mu^{*} \in \mathbb{C}^{*}$ which we will use quite often. The above inclusion (a) shows that the complex $N$-inner product is linear in the first factor. Since in case (c) we have complex conjugates $\overline{\lambda^{*}}$ and $\overline{\mu^{*}}$ on the right, we say that the complex $N$ inner product is conjugate linear (semilinear) in the second factor.

Following Çakmak and Başar [7] we can obtain by a simple straightforward calculation that an $\alpha$-norm on a real
$N$-inner product space satisfies the fact that the important equality

$$
\|x \dot{+} y\|_{N}^{2_{N}} \dot{+}\|x \dot{-} y\|_{N}^{2_{N}}=2 \dot{x}\left(\|x\|_{N}^{2_{N}} \dot{+}\|y\|_{N}^{2_{N}}\right)
$$

holds for all $x, y \in \mathbb{R}(N)$. Similarly, it is trivial that a $*$-norm on a complex $N$-inner product space satisfies the equality

$$
\ddot{\| x} \hat{+} y\left\|^{2} \ddot{+} \ddot{\|} x \hat{=} y\right\|^{2}=2 \ddot{\times}\left(\ddot{\|} x\left\|^{2} \ddot{+} \ddot{\|} y\right\|^{2}\right)
$$

which holds for all $x, y \in \mathbb{C}^{*}$.

Example 29. The following examples can be given as follows.

(1) In the previous section, we give in some details the non-Cartesian plane (*-plane) as an example of a $N$ vector space.

(2) Euclidian space: the space $\mathbb{R}^{n}(N)$ is a Hilbert space with real $N$-inner product defined by

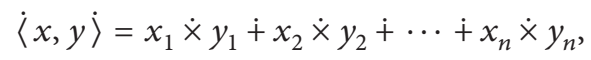

where $x=\left(x_{1}, x_{2}, \ldots, x_{n}\right), y=\left(y_{1}, y_{2}, \ldots, y_{n}\right) \in$ $\mathbb{R}^{n}(N)$. In fact, from (28), we obtain

$$
\|x\|_{N}=\sqrt{\langle x, x\rangle}^{N}={\sqrt{x_{1}^{2_{N}}+x_{2}^{2_{N}}+\cdots \dot{+} x_{n}^{2_{N}}}}^{N} .
$$

(3) Unitary Space; the space $\mathbb{C}^{* n}$ is a Hilbert space with complex $N$-inner product given by

$$
\ddot{\langle} x, y \ddot{\rangle}=x_{1} \widehat{\times} \overline{y_{1}} \hat{+} x_{2} \widehat{\times} \overline{y_{2}} \hat{+} \cdots \widehat{+} x_{n} \widehat{\times} \overline{y_{n}} .
$$

In fact, from (30), we obtain the *-norm defined by

$$
\begin{aligned}
& \ddot{\|} \ddot{x}=\sqrt{\ddot{\langle x, x \ddot{\rangle}}}=\sqrt{x_{1} \widehat{\times} \overline{x_{1}} \hat{+} \cdots \hat{+} x_{n} \widehat{\times} \overline{x_{n}}}
\end{aligned}
$$

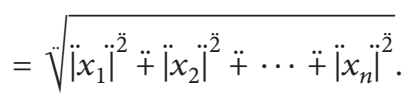

(4) Sequence Space: by taking into account $*$-sum with complex terms in (18), the space $\ell_{2}^{*}$ in Section 3.2, is a Hilbert space with $N$-inner product defined by

$$
\ddot{\langle} x, y \ddot{\rangle}=\sum_{i=1}^{\infty} x_{i} \widehat{\times} \overline{y_{i}}=x_{1} \widehat{\times} \overline{y_{1}} \hat{+} x_{2} \widehat{\times} \overline{y_{2}} \hat{+} \ldots \hat{+} x_{i} \widehat{\times} \overline{y_{i}} \hat{+} \cdots,
$$

and since the $*$-convergence of this series is straightforward we omit the details.

Lemma 30. Let $X$ be a complex $N$-inner product space. For all $a, b, c \in X$ and $\lambda, \mu \in \mathbb{C}^{*}$. Then

(i) $\left\langle\theta^{*}, b\right\rangle=\left\langle a, \theta^{*}\right\rangle=\theta^{*}$;

(ii) $\langle a, \lambda \hat{\times} b \hat{+} \mu \widehat{\times} c\rangle=\bar{\lambda} \hat{\times}\langle a, b\rangle \hat{+} \bar{\mu} \hat{\times}\langle a, c\rangle$; 
(iii) let $\alpha$ and $\beta$ be the same generator. Then $\ddot{\langle} \lambda \hat{\times} a \widehat{+} \mu \widehat{\times} b, \lambda \hat{\times} a \widehat{+} \mu \widehat{\times} b \ddot{\rangle}$ can be evaluated as

$$
\begin{aligned}
& \left.\left.\ddot{|\lambda|^{2}} \widehat{x} \ddot{\langle a} a, a\right\rangle \hat{+}(\lambda \hat{\times} \bar{\mu}) \widehat{\times} \ddot{\langle a} a, b\right\rangle \\
& \widehat{+}(\mu \widehat{\times} \bar{\lambda}) \widehat{\times} \ddot{\langle} b,\left.a \ddot{\rangle} \widehat{+} \ddot{\mid \mu}\right|^{2} \widehat{\times} \ddot{\langle} b, b \ddot{\rangle},
\end{aligned}
$$

where the modulus function $\ddot{\mid} \lambda \ddot{\mid}^{2}=\lambda \hat{\times} \bar{\lambda}$ and $\ddot{\mid} \mu \ddot{\mid}^{2}=$ $\mu \widehat{\times} \bar{\mu}$.

Proof. For $a, b, c \in X$ and $\theta^{*}, \lambda, \mu \in \mathbb{C}^{*}$, by the using complex conjugate in Definition 11, then

(i) $\left\langle\theta^{*}, b\right\rangle=(\dot{0}, \ddot{0}) \widehat{\times}(\dot{b}, \ddot{-} \ddot{b})=(\dot{a}, \ddot{-} \ddot{a}) \widehat{\times}(\dot{0}, \ddot{0})=\left\langle a, \theta^{*}\right\rangle=$ $\theta^{*}$;

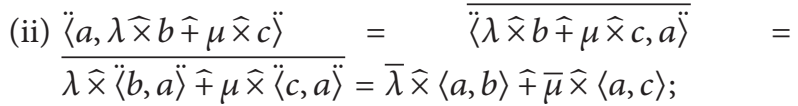

(iii) suppose that $\alpha$ and $\beta$ are the same, then, the $N$-inner product $\ddot{\langle} \lambda \widehat{\times} a \hat{+} \mu \widehat{\times} b, \lambda \hat{\times} a \hat{+} \mu \widehat{\times} b \ddot{\rangle}$ can be rewritten as $\quad \bar{\lambda} \hat{\times} \ddot{\langle\lambda} \hat{\times} a \hat{+} \mu \hat{\times} b, a\rangle \hat{+} \bar{\mu} \hat{\times} \ddot{\langle\lambda} \hat{\times} a \hat{+} \mu \hat{\times} b, b\rangle$ $=(\lambda \hat{\times} \bar{\lambda}) \hat{x} \ddot{\langle} a, a \ddot{\gamma} \hat{+}(\bar{\lambda} \hat{\times} \mu) \hat{x} \ddot{\langle} b, a \ddot{\gamma} \hat{+}(\bar{\mu} \hat{\times} \lambda) \hat{\times} \ddot{\langle} a, b \ddot{\rangle}$

$\hat{+}(\mu \widehat{x} \bar{\mu}) \hat{x} \ddot{\langle} b, b \ddot{\rangle}$. This completes the proof.

First of all, we should verify that (24) in the preceding section defines a $*$-norm: (N1) and (N2) in Definition 18 follow from (CIP4). Furthermore, (N3) is obtained by the use of (CIP2) and (CIP3); in fact $\ddot{\|} \lambda \hat{\times} x \ddot{\|}=\sqrt{\ddot{\langle} \lambda \widehat{\times} x, \lambda \widehat{\times} x \ddot{\rangle}}=$ $\sqrt{\lambda \hat{\times} \bar{\lambda}} \ddot{\times} \sqrt{\ddot{\langle x} x, x\rangle}=\ddot{\mid} \lambda \ddot{\mid} \ddot{\times} \ddot{\|} x \ddot{\|}$. Finally, (N4) is included in the following.

Lemma 31. A N-inner product and the corresponding norm satisfy the Cauchy-Schwarz inequality and the triangle inequality as follows.

(a) Cauchy-Schwarz: one has

$\dddot{\mid}\langle x, y\rangle \ddot{\mid} \ddot{\leq} \ddot{\|} x \ddot{\|} \ddot{x} \ddot{\|} y \ddot{\|}\left(\right.$ for real case $\left.\left.|\dot{\langle} x, y\rangle\right|_{N} \dot{\leq}\|x\|_{N} \dot{\times}\|y\|_{N}\right)$,

where the equality sign holds if and only if $\{x, y\}$ is a linearly dependent set.

(b) Triangle inequality: one has

$\ddot{\|} x \hat{+} y \| \ddot{\leq} \ddot{\|} x \ddot{\|} \ddot{\|} y \ddot{\|}\left(\right.$ for real case $\left.\|x \dot{+} y\|_{N} \dot{\leq}\|x\|_{N} \dot{+}\|y\|_{N}\right)$,

where the equality sign holds if and only if $y=\theta^{*}$ or $x=c \widehat{\times} y$ where $c \in \mathbb{R}(N)$.

Proof. Since the proof of real parts is trivial then we only show the complex parts. (a) If $y=\theta^{*}$, then (34) holds. Let $y \neq \theta^{*}$. From the part (iii) of Lemma 30, for every scalar $\lambda$ we have

$$
\begin{aligned}
& \ddot{0} \ddot{\leq} \ddot{\| x}=\lambda \hat{\times} y \|^{2}=\ddot{\langle} x \hat{-} \lambda \hat{x} y, x=\lambda \hat{x} y \ddot{\rangle} \\
& =\ddot{\langle x}, x\rangle=\bar{\lambda} \hat{x} \ddot{\langle} x, y\rangle=\lambda \hat{x}[\ddot{\langle} y, x\rangle=\bar{\lambda} \hat{x} \ddot{\langle} y, y\rangle] \text {. }
\end{aligned}
$$

We see that the expression in above brackets is $\theta^{*}$ if we choose $\lambda=\ddot{\langle} y, x \ddot{x} \ddot{\|}\langle y, y\rangle$. The remaining inequality is

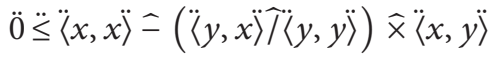

$$
\begin{aligned}
& =\ddot{\| x} \ddot{\|}^{2}=\left[\dddot{i}\langle x, y\rangle \ddot{\mid}^{2} \ddot{\gamma} \ddot{\|} y \ddot{\|}^{2}\right],
\end{aligned}
$$

and hence $\left.\dddot{\imath}\langle x, y\rangle \ddot{\mid}^{2} \ddot{\leq} \ddot{\langle} x, x \ddot{x} \ddot{x} \ddot{\langle} y, y\right\rangle$; here we used $\ddot{\langle} y, x\rangle=\ddot{\langle} x, y\rangle$. Equality holds in this derivation if and only if $y=\theta^{*}$; hence $x \widehat{-} \lambda \hat{\times} y=\theta^{*}$, so that $x=\lambda \widehat{\times} y$, which shows linear dependence.

(b) We have

$\left.\ddot{\| x} \widehat{+} y \ddot{\|}^{2}=\ddot{\langle} x \widehat{+} y, x \widehat{+} y \ddot{\rangle}=\ddot{\|} x \ddot{\|}^{\ddot{+}} \ddot{+} \ddot{\langle} x, y\right\rangle \ddot{+} \ddot{\langle} y, x \ddot{\rangle} \ddot{+} \ddot{\|} y \|^{2}$.

By the Cauchy-Schwarz inequality, $\dddot{\mid}\langle x, y\rangle \mid=$

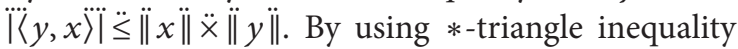
in Lemma 19(i), we thus obtain

$$
\begin{aligned}
& \left.\ddot{\| x} \hat{+} y \ddot{\|}^{\ddot{2}} \ddot{\leq} \ddot{\|} x \ddot{\|}^{2} \ddot{+} \ddot{2} \ddot{x} \ddot{\mid} x, y\right\rangle \mid \ddot{+} \ddot{\|} y \ddot{\|}^{2} \\
& \ddot{\leq} \ddot{\|} x \ddot{\|}^{\ddot{2}} \ddot{+} \ddot{2} \ddot{\times} \ddot{\|} x \ddot{\|} \ddot{x} \ddot{\|} y \ddot{\|} \ddot{+} \ddot{\|} y \|^{2}=(\ddot{\|} x \ddot{\|} \ddot{+} \ddot{\|} y \|)^{2}
\end{aligned}
$$

Taking $\beta$-square roots on both sides, we have (35). Equality holds in this derivation if and only if $\ddot{\langle} x, y \ddot{\rangle} \ddot{+} \ddot{\langle} y, x \ddot{\rangle}=\ddot{2} \ddot{\times} \dddot{\mid}\langle x, y\rangle \mid \overrightarrow{\mid}$. The left hand side is $2 \mathscr{R} e \ddot{\langle} x, y\rangle$ where $\mathscr{R} e$ denotes the real part. From this and the inclusion (34), we obtain

$$
\mathscr{R} e \ddot{\langle} x, y \ddot{\rangle}=\ddot{\|} x \ddot{\|} \ddot{x} \ddot{\|} \| \ddot{\geq} \ddot{\mid}\langle x, y\rangle \ddot{\mid} \text {. }
$$

Since the real part of a non-Newtonian complex number cannot exceed the absolute value, we must have equality, which implies linear dependence by part (a), say, $y=\theta^{*}$ or $x=c \hat{x} y$. We show that $c \in \mathbb{R}(N)$. From (40) with the equality sign we have $\mathscr{R} \ddot{\langle} x, y \ddot{\rangle}=\dddot{\mid} \ddot{\langle} x, y\rangle \ddot{\mid}$. But if the real part of a non-Newtonian complex number equals the absolute value, the imaginary part must be $\ddot{0}$. Hence $\dddot{\mid}\langle x, y\rangle \mid=\mathscr{R} e \ddot{\langle} x, y \ddot{\rangle} \dot{0}$ by (40), and $c \dot{0} \dot{0}$ follows from $\ddot{0} \ddot{\leq} \ddot{\langle} x, y \ddot{\rangle}=\ddot{\langle} c \hat{\times} y, y\rangle=c \ddot{\times} \ddot{\|} y \ddot{\|}^{2}$.

Since every $\mathrm{N}$-inner product has an induced corresponding norm, a natural question is whether every nonNewtonian norm is induced by a $N$-inner product. The answer is no, because non-Newtonian norms induced by a $N$ inner products have some special properties that these norms in general do not possess. 
Lemma 32. Let $X$ be a complex $N$-inner product space and suppose that $\left\{x_{n}\right\}$ and $\left\{y_{n}\right\}$ are *-convergent sequence with complex terms in $X$, with ${ }^{*} \lim _{n \rightarrow \infty} x_{n}=x$ and ${ }^{*} \lim _{n \rightarrow \infty} y_{n}=y$. Then $\left.{ }^{*} \lim _{n \rightarrow \infty} \ddot{\langle} x_{n}, y_{n} \ddot{\rangle}=\ddot{\langle} x, y\right\rangle$.

Proof. Consider the following:

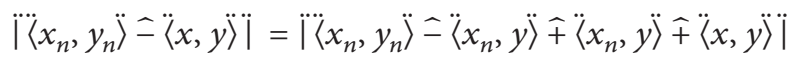

$$
\begin{aligned}
& \left.\left.\ddot{\leq} \ddot{\mid}\left\langle x_{n}, y_{n} \ddot{\rangle}=\ddot{\langle} x_{n}, y\right\rangle \ddot{\mid} \ddot{+} \dddot{\mid} \ddot{\langle} x_{n}, y\right\rangle=\ddot{\langle} x, y\right\rangle \ddot{\mid} \\
& =\dddot{\mid}\left\langle x_{n}, y_{n} \hat{-} y\right\rangle \ddot{\mid} \ddot{+} \dddot{\mid}\left\langle x_{n} \hat{-} x, y\right\rangle \ddot{\mid}
\end{aligned}
$$

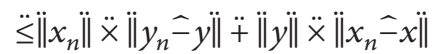

Since the sequence $\left\{x_{n}\right\}$ is $*$-convergent, $\ddot{\|} x_{n} \ddot{\|}$ is $*$-bounded, so the right-hand side of this inequality tends to $\ddot{0}$ as $n \rightarrow \infty$. This completes the proof.

We finally mention the following interesting fact. We know that for a complex $N$-inner product there corresponds a norm which is given by (24). Conversely, we can rewrite the complex $N$-inner product from the corresponding norm. In fact, one may verify by straightforward calculation that for a real $N$-product space we have $\langle x, y\rangle=$ $\left(\|x \dot{+} y\|_{N}^{2_{N}}-\|x \dot{-} y\|_{N}^{2_{N}}\right) \dot{/ 4}$ and for a complex $N$-inner product space we have $\mathscr{R} e \ddot{\langle} x, y\rangle=\left(\ddot{\|} x \hat{+} y\left\|^{\ddot{2}} \ddot{-} \ddot{\|} x \hat{-} y\right\|^{\ddot{2}}\right) \ddot{/} \ddot{4}$ and $\mathscr{I} m\langle x, y\rangle=\left(\ddot{\|} z\left\|^{\ddot{2}} \ddot{-} \ddot{\|} \bar{z}\right\|^{2}\right) \ddot{/} \ddot{4}$ for all $z=(\dot{x}, \ddot{y}) \in \mathbb{C}^{*}$.

\section{Concluding Remarks}

Although all arithmetics are isomorphic, only by distinguishing among them do we obtain suitable tools for constructing all the non-Newtonian calculi. But the usefulness of arithmetics is not limited to the construction of calculi; we believe there is a more fundamental reason for considering alternative arithmetics; they may also be helpful in developing and understanding new systems of measurement that could yield simpler physical laws.

Consider the concept of the average speed. The definition "distance traveled per unit time" is incomplete because it fails to provide a method of determining the average speed of an accelerated particle. The definition "distance divided by time," though not incorrect, is a gross oversimplification that fails to reveal the underlying issues. Fortunately there is a completely satisfactory definition, which undoubtedly was known to Galileo. Then we isolate a constant in each given uniform motion by defining speed to be the distance traveled in any unit time-interval. Finally, for a particle that moves nonuniformly a distance $d$ in time $t$, we define the average speed to be the speed that a particle in uniform motion must have in order to travel a distance $d$ in time $t$. In our opinion, neither the simplicity nor the obviousness of the answer, $d / t$, justifies its use as the definition of average speed. The critical step in defining average speed is the isolation of a constant (speed) in the phenomenon of uniform motion. Similarly, the critical step in defining the $*$-gradient is the identification of a constant (*-slope) for any given power function [2].
The theory of Hilbert spaces does not deal with angles in general. But the presence of the additional algebraic structures of inner product greatly enriches the geometric properties of the space. Most significantly, it enables us to introduce a notion of perpendicularity for two vectors and the geometry corresponds in several fundementals respects with Euclidean geometry in classical mean. In particular, this leads to the important projection theorems, a generalized theory of Fourier series, a most sensible definition of an operator, and a powerful body of the theory based on this new concept. By using the above-mentioned ideas, one can conclude that the study gives us a new tool about inner product spaces and Hilbert spaces via $*$-calculus.

As a natural continuation of this paper, we should record from now on that it is meaningful to define non-Newtonian trigonometric structures on non-Cartesian plane and study the notion of orthogonality with respect to the $*$-calculus.

\section{Conflict of Interests}

The authors declare that there is no conflict of interests regarding the publication of this paper.

\section{References}

[1] M. Grossman, Bigeometric Calculus, Archimedes Foundation Box 240, Rockport, Massachusetts, Mass, USA, 1983.

[2] M. Grossman and R. Katz, Non-Newtonian Calculus, 1978.

[3] M. Grossman, The First Nonlinear System of Differential and Integral Calculus, 1979.

[4] A. E. Bashirov, E. M. Kurpınar, and A. Özyapıcı, "Multiplicative calculus and its applications," Journal of Mathematical Analysis and Applications, vol. 337, no. 1, pp. 36-48, 2008.

[5] A. Bashirov and M. Riza, "On complex multiplicative differentiation," TWMS Journal of Applied and Engineering Mathematics, vol. 1, no. 1, pp. 75-85, 2011.

[6] A. Uzer, "Multiplicative type complex calculus as an alternative to the classical calculus," Computers and Mathematics with Applications, vol. 60, no. 10, pp. 2725-2737, 2010.

[7] A. F. Çakmak and F. Başar, "Certain spaces of functions over the field of non-Newtonian complex numbers," Abstract and Applied Analysis, vol. 2014, Article ID 236124, 12 pages, 2014.

[8] S. Tekin and F. Basar, "Certain sequence spaces over the nonNewtonian complex field," Abstract and Applied Analysis, vol. 2013, Article ID 739319, 11 pages, 2013.

[9] L. Florack and H. van Assen, "Multiplicative calculus in biomedical image analysis," Journal of Mathematical Imaging and Vision, vol. 42, no. 1, pp. 64-75, 2012.

[10] S. L. Blyumin, "Discreteness versus continuity in information technologies: quantum calculus and its alternatives," Automation and Remote Control, vol. 72, no. 11, pp. 2402-2407, 2011.

[11] U. Kadak, "Determination of the Köthe-Toeplitz duals over the non-Newtonian complex field," The Scientific World Journal, vol. 2014, Article ID 438924, 10 pages, 2014.

[12] U. Kadak and H. Efe, "Matrix transformations between certain sequence spaces over the non-Newtonian complex field," The Scientific World Journal, vol. 2014, Article ID 705818, 2014. 
[13] E. Misirli and Y. Gurefe, "Multiplicative Adams BashforthMoulton methods," Numerical Algorithms, vol. 57, no. 4, pp. 425-439, 2011.

[14] A. F. Çakmak and F. Başar, "Some new results on sequence spaces with respect to non-Newtonian calculus," Journal of Inequalities and Applications, vol. 2012, article 228, 2012. 


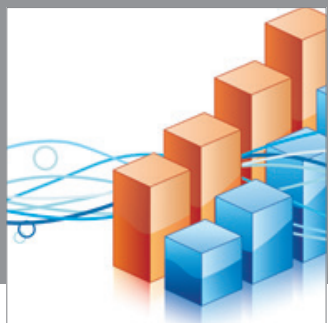

Advances in

Operations Research

mansans

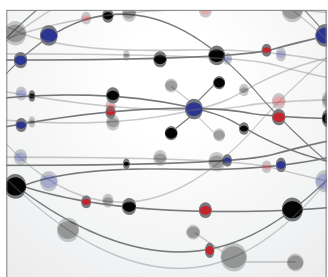

The Scientific World Journal
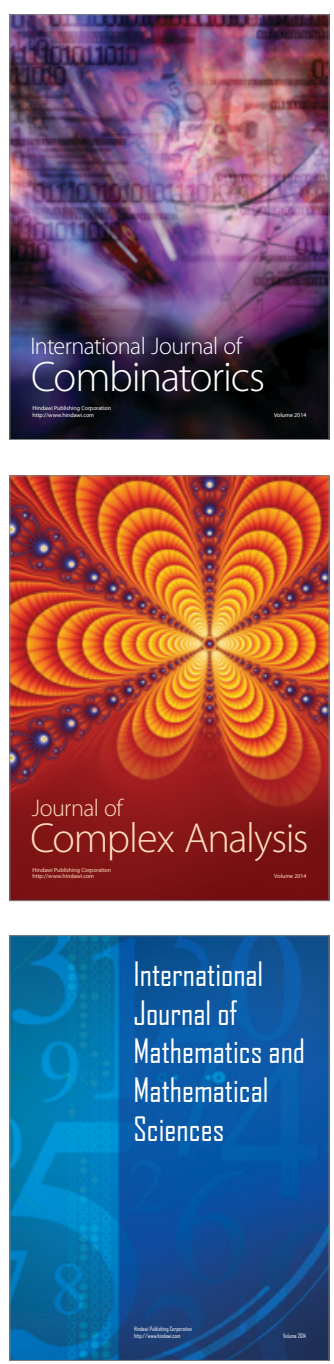
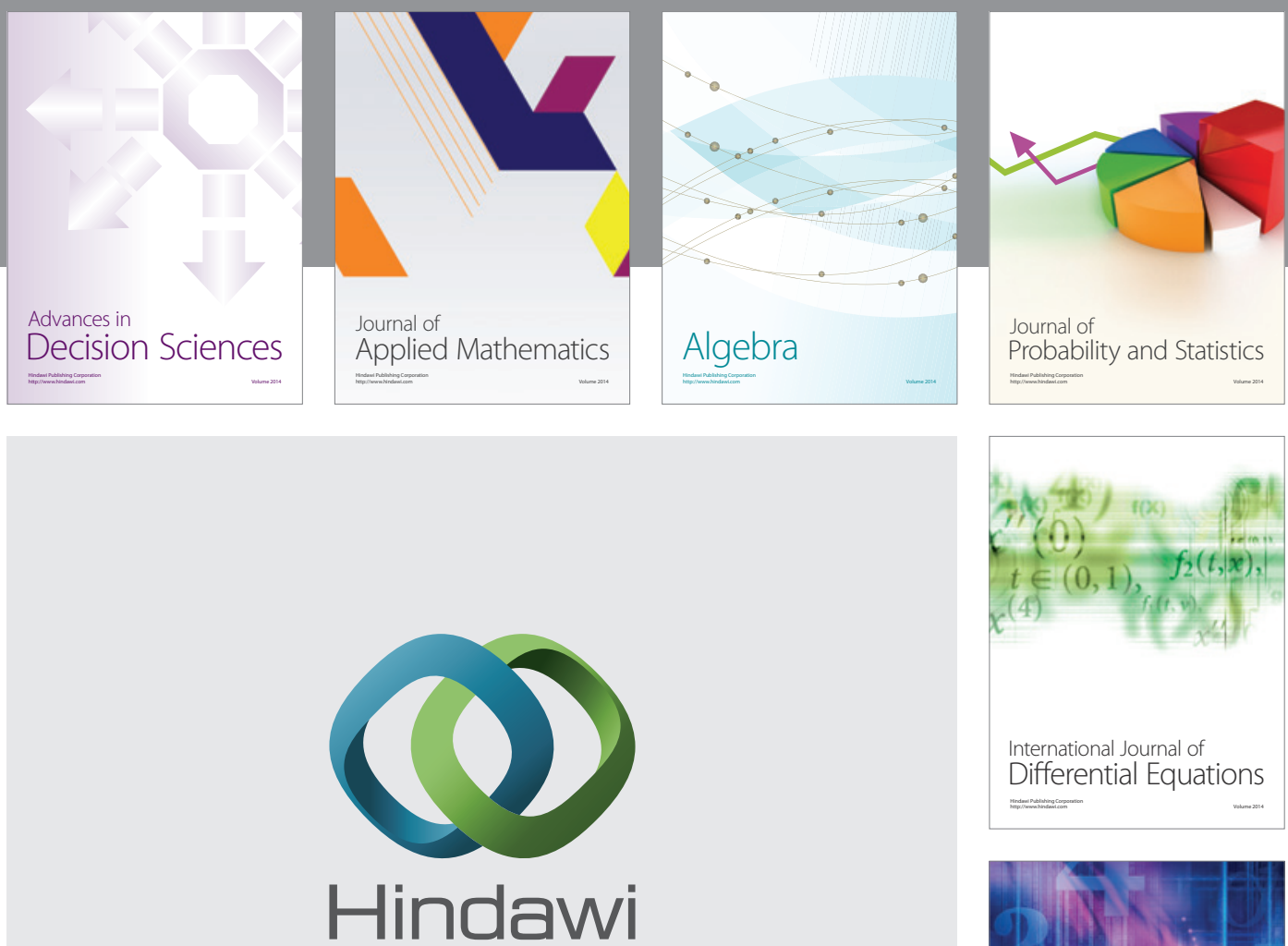

Submit your manuscripts at http://www.hindawi.com
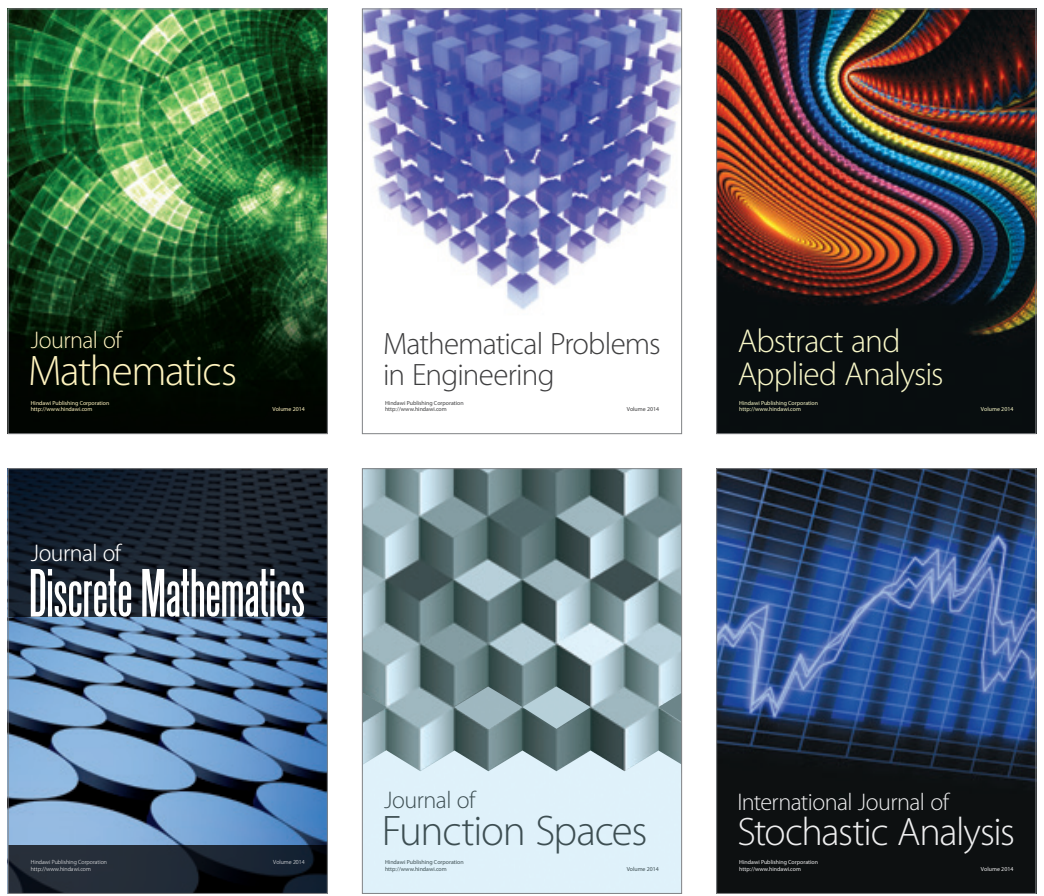

Journal of

Function Spaces

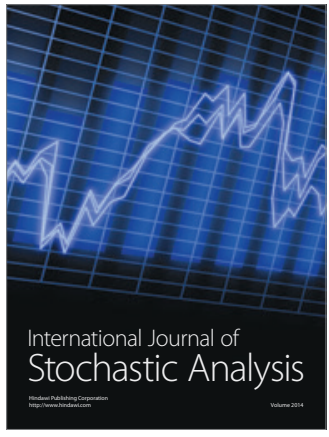

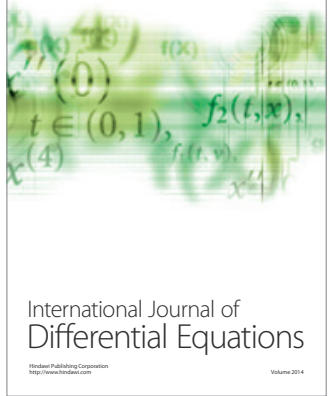
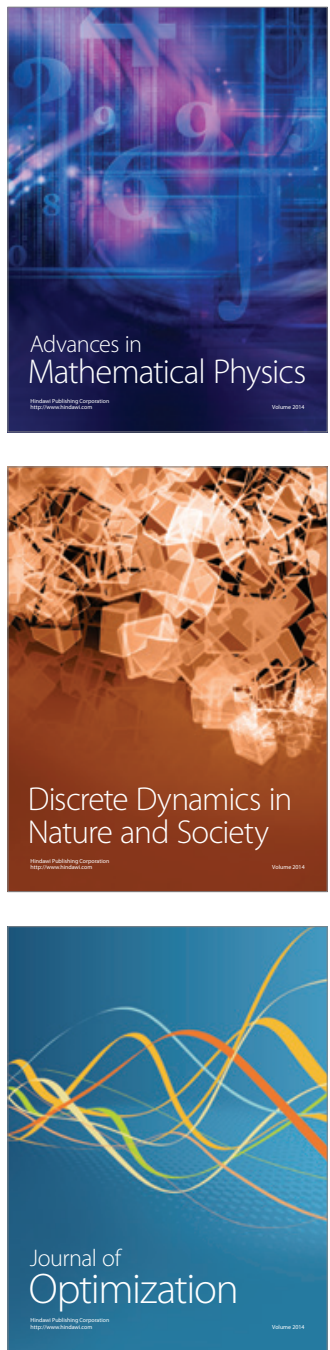